Recherches en didactique des langues et des cultures

Les cahiers de l'Acedle

10-3 | 2013

Enseignements universitaires francophones en milieux bi / plurilingues

\title{
Enseignements universitaires francophones en milieux bi / plurilingues : légitimations et mises
} en œuvre

Présentation du numéro

Danielle Omer et Frédéric Tupin

\section{(2) OpenEdition}

Journals

Édition électronique

URL : http://journals.openedition.org/rdlc/2628

DOI : $10.4000 /$ rdlc. 2628

ISSN : $1958-5772$

Éditeur

ACEDLE

Référence électronique

Danielle Omer et Frédéric Tupin, « Enseignements universitaires francophones en milieux bi /

plurilingues : légitimations et mises en œuvre », Recherches en didactique des langues et des cultures

[En ligne], 10-3 | 2013, mis en ligne le 27 décembre 2013, consulté le 01 mai 2019. URL : http://

journals.openedition.org/rdlc/2628; DOI : 10.4000/rdlc.2628

Ce document a été généré automatiquement le 1 mai 2019.

\section{cc) (†) $\ominus$}

Recherches en didactique des langues et des cultures is licensed under a Creative Commons AttributionNonCommercial-NoDerivatives 4.0 International License 


\section{Enseignements universitaires francophones en milieux bi / plurilingues : légitimations et mises en œuvre}

Présentation du numéro

Danielle Omer et Frédéric Tupin

\section{Sur la thématique du dossier}

1 Le dossier thématique de ce numéro est consacré à des problématiques sociolinguistiques et didactiques propres à des dispositifs où l'enseignement universitaire en français est proposé - quelquefois imposé - par un nombre bien déterminé d'établissements spécifiques (certaines facultés, des écoles ou des instituts supérieurs) à des étudiants dont ce n'est pas la langue première et qui n'ont été que partiellement, voire jamais, scolarisés en français.

2 Plus exactement ce sont les légitimations de ces dispositifs et les conditions de leurs mises en œuvre qui font l'objet des sept études rassemblées ici. Elles sont analysées à travers des perspectives qui sont, le plus souvent, représentatives du propre positionnement des auteurs au sein de ces dispositifs. En effet, non seulement ceux-ci entretiennent des rapports étroits à l'objet de leur étude par le fait qu'ils sont fortement engagés par les méthodes de recherches ad hoc qu'ils ont construites afin d'enquêter sur les terrains de ces établissements universitaires observés et analysés. Mais, de plus, ils bénéficient de connaissances complémentaires dues au fait que la plupart d'entre eux sont (ou ont été) des enseignants de langue française dans ces formations, ou bien encore exercent (ou ont exercé) des fonctions de coordination ou d'encadrement dans ces institutions. À ce titre, ils en connaissent de l'intérieur à la fois le fonctionnement et les acteurs, ce qui oriente, en les complétant, les données recueillies et va bien au-delà des simples connaissances obtenues grâce à une méthode d'enquête externe. On peut objecter 
qu'il s'agit là de paramètres qui accroissent la subjectivité dans les recherches entreprises puisqu'il y a un engagement personnel de la part du chercheur. De notre point de vue, cette subjectivité, inhérente à toute recherche, peut se réduire dès lors qu'elle est clairement circonscrite et rendue visible ce qui, d'une certaine façon, revient à «l'objectiver». En conséquence, nous pensons que de telles précisions, clairement annoncées dans cette présentation, comme dans les articles, donnent aux lecteurs les moyens de comprendre l'origine des points de vue adoptés dans chacune des études et d'en apprécier les raisons ce qui, au bout du compte, leur permet aussi de construire leurs propres points de vue en toute connaissance de cause.

\section{Présentation des contributions du dossier thématique}

3 Les deux contributions, qui ouvrent le dossier et s'éclairent mutuellement, portent sur des aspects de l'acquisition de compétences bi / plurilingues à base francophone dans un environnement où le français, langue minoritaire de la province (en Ontario, au Canada), est co-langue officielle avec l'anglais au niveau de l'État. Tout d'abord Hélène Knoerr et Alysse Weinberg, dans leur article intitulé L'immersion à l'Université d'Ottawa: une innovation héritée du passé, proposent un historique du « régime d'immersion en français » à l'Université d'Ottawa. Il s'agit d'une filière qui permet aux étudiants anglophones de poursuivre, de manière encadrée et sous certaines conditions leurs études en français. À la suite de cet historique, elles détaillent l'organisation actuelle du régime d'immersion et exposent brièvement les recherches auxquelles il donne lieu. En écho à ce premier article à la tonalité claire et positive, celui de Julie Byrd Clark, Sylvie Lamoureux et Sofia Stratilaki, intitulé Apprendre et enseigner le français dans l'Ontario, Canada: entre dualité linguistique et réalités plurielles complexes questionne la politique linguistique officielle du gouvernement canadien, qui, en favorisant un «monolinguisme dédoublé» (anglais canadien et français québécois ou de France), rend plus difficile la légitimation du français ontarien ainsi que celle du français des "francophones par choix », et, au bout du compte, la légitimation des contextes plurilingues complexes.

4 Les deux contributions qui suivent portent sur le français utilisé comme langue d'enseignement dans certaines filières universitaires de deux pays qui ne reconnaissent que l'arabe et l'amazighe comme langues officielles ${ }^{2}$ de l'État, à savoir le Maroc et l'Algérie. Dans la première, intitulée L'impact du choix de la langue sur les débouchés et carrières professionnels: points de vue d'étudiants, Abdellatif Bensfia, Abdelouahed Mabrour et Khalil Mgharfaoui montrent que le français, en théorie langue étrangère, est une langue dont la maîtrise est essentielle dans de très nombreux secteurs du marché de l'emploi marocain, tout en étant "objet d'un enjeu idéologique » entre les tenants de la tradition arabo-islamique et les partisans de la modernité. Une enquête qualitative menée dans la faculté de droit à l'université Hassan II, à Casablanca, et dans l'école de journalisme à l'Isic de Rabat, où il est possible, dans les deux cas, de choisir de faire ses études soit dans une section arabophone soit dans une section francophone, confirme que le choix de la section linguistique par les étudiants se fait surtout en fonction des compétences linguistiques personnelles même si une majorité des enquêtés semble reconnaître que les sections francophones offrent de meilleurs débouchés professionnels. Ce constat renvoie à la question des stratégies choisies pour l'enseignement à l'école des (et dans les) langues concernées, dont même le ministère de tutelle reconnaît l'indigence. 
Dans le deuxième article intitulé Français langue d'enseignement et prise en compte du bi/ plurilinguisme dans les études universitaires en Algérie: quelles compatibilités avec la didactique $d u$ FOS ? Claude Cortier, Abdelkrim Kaaboub, Nawel Kherra et Mahieddine Benaoum, après avoir rappelé que le français en Algérie a officiellement le statut d'une langue étrangère parmi d'autres mais qu'il est «langue d'enseignement et langue de rédaction des travaux académiques dans les filières universitaires médicales, scientifiques et techniques et en architecture ", soulignent que les étudiants s'inscrivant dans ces filières sont débutants en français pour $80 \%$ d'entre eux, ce qui entraîne un taux d'échec très important en première année. Les auteurs rendent compte de leurs recherches-actions qui portent sur quelques filières en sciences physiques (Alger), sciences agro-vétérinaires (Blida), sciences économiques (Mascara) et architecture (Sétif). À partir d'observations directes, d'analyses de corpus de cours enregistrés et d'entretiens avec les étudiants et les enseignants, ils décrivent et analysent les pratiques déclarées et les pratiques observées des uns et des autres concernant la prise de notes et la compréhension des cours du côté des étudiants, la tenue des cours magistraux et la formulation des évaluations écrites par les enseignants. Une ingénierie de formation, à la demande des établissements, a été mise en place afin de donner aux étudiants des outils pour être plus à l'aise avec le français mais aussi pour veiller à ce que le plurilinguisme existant (arabe dialectal, arabe standard, français et tamazight selon les régions) perdure durant les études et se consolide loin de l'insécurité linguistique dominante au sein des filières universitaires.

6 Les deux articles suivants ont pour objet d'étude deux établissements universitaires atypiques (au Caire, en Egypte et à Istanbul, en Turquie), où le français est (co)-langue d'enseignement. Le Caire et Istanbul sont deux villes où le français, sans avoir jamais été une langue de colonisation, fut très présent au $\mathrm{xIX}^{\mathrm{e}}$ siècle comme langue de communication véhiculaire entre les communautés des minorités religieuses ${ }^{3}$ et linguistiques des Arméniens, des Grecs, des Juifs, etc. (Calvet, 2003) et comme langue européenne des élites locales de langue première respectivement arabe ou turque. Le français était par ailleurs, en partie, langue de travail dans certaines administrations. À partir du dernier tiers du XIX ${ }^{\mathrm{e}}$, de très nombreuses écoles françaises, confessionnelles la plupart du temps, contribuèrent à la diffusion du français dans ces deux pays. Ensuite, au cours $\mathrm{du} \mathrm{xx}^{\mathrm{e}}$ siècle après la révolution kémaliste pour Istanbul (Aksöy, 2007), et après l'indépendance puis la révolution nassérienne pour Le Caire (Abécassis, 2006), le français perdit peu à peu son rôle de premier plan et son prestige. À présent, il n'est le plus souvent qu'une LV2 à l'école, après l'anglais. Cependant en raison de cette histoire et des représentations positives qui l'accompagnent, le français dispose encore, auprès d'un certain public lettré, d'un capital qui a permis l'implantation de deux établissements francophones: l'Université française d'Egypte, au Caire et l'Université Galatasaray, à Istanbul.

Dans sa contribution intitulée Représentations et pratiques linguistiques des étudiants de la faculté des Langues appliquées de l'Université française d'Égypte et de leur famille, Catherine Julien-Kamal s'attache à analyser les représentations et les pratiques langagières dans les contextes familiaux des étudiants (public féminin à plus de $95 \%$ ) de la faculté des Langues appliquées de l'UFE, au Caire. Grâce à des questionnaires distribués aux parents et aux étudiants ainsi que des biographies langagières rédigées par ces derniers, l'auteure constate que le choix d'une bonne partie d'entre eux est largement motivé par les études bilingues arabe / français d'un ou des deux parents, la mère très fréquemment. De plus, presque les deux tiers du public enquêté déclarent utiliser au moins deux langues à la 
maison (arabe / français ou arabe / anglais) voire même quelquefois trois. Dans un nombre de cas significatif, les membres de la fratrie suivent des formations bilingues selon leur appartenance sexuelle (français pour les filles et anglais pour les garçons). On note aussi, et entre autres, que les étudiantes ne reprennent pas à leur compte l'idée largement répandue que le français est une langue pour les filles, mais préfèrent parler d'une langue difficile et élitiste. Au bout du compte, il semble que beaucoup de familles concernées cherchent avant tout à maintenir le bi /plurilinguisme familial et au-delà, l'appartenance au cercle restreint d'une élite lettrée, dont le français serait la marque.

8 De son côté, Christel Troncy cherche à comprendre, dans son étude intitulée Sociogenèse de départements en langue française à Istanbul, Bucarest et Sofia au tournant des années 1990. Entre petites et grandes politiques linguistiques universitaires, les raisons et les circonstances d'un projet d'envergure: la création de l'Université Galatasaray à Istanbul. Selon elle, cette université francophone pour les sciences politiques, les relations internationales, la gestion, est représentative de beaucoup d'autres sections universitaires francophones, généralement plus modestes, créées à cette époque, dans des pays où le français n'est ni langue locale, ni nationale, ni officielle ni, non plus, langue de scolarisation. Elle construit alors des objets de comparaison en incluant des créations datant de la même époque mais beaucoup plus modestes, à Bucarest (en Roumanie) et à Sofia (en Bulgarie), deux pays qui, soit ont fait partie de l'Empire ottoman, la Bulgarie, soit ont entretenu des liens de vassalité avec lui, la Roumanie. Il se trouve également que ces trois pays ont cherché, dans les années 1990 mais, dans des circonstances différentes, à se rapprocher de ce qui est maintenant devenu l'Union européenne. Au terme de sa démarche analytique appuyée sur des documents d'archives et des entretiens avec les divers responsables concernés, Christel Troncy montre que ce sont, avant tout, les acteurs locaux et moins les acteurs de la francophonie institutionnelle qui ont été moteurs dans le projet de création de l'Université Galatasaray comme dans les sections universitaires francophones de Bucarest et Sofia. Bien que le choix du français comme langue d'enseignement n'ait pas été immédiatement consensuel, loin s'en faut, il a permis d'établir un partenariat avec des associés puissants (le MAE français et aussi l'Aupelf, un peu plus tardivement). C'est à ce titre-là surtout que le choix du français a convaincu et non pas au nom de la défense d'un bi / plurilinguisme, ce qui n'est pas sans conséquence.

Le dossier thématique se clôt par la contribution de Marc Debono, Emmanuelle Huver et Céline Peigné intitulée Figures et traitements de la diversité. Vers une diversité diversitaire en didactiques des langues? qui questionne la notion de "diversité » souvent utilisée en didactique des langues en général, quand le sujet des variétés langagières, des publics d'apprenants et des dispositifs de formation est abordé. Il s'agit, en particulier, d'une notion phare en didactique du bi / plurilinguisme, dès lors qu'elle permet la prise en compte de certaines pluralités linguistiques et culturelles sans toutefois aller, selon les auteurs, jusqu'à celles qui sont imbriquées et complexes dont traite, par exemple, l'article de Julie Byrd Clark, Sylvie Lamoureux et Sofia Stratilaki dans ce dossier. Pour Marc Debono, Emmanuelle Huver et Céline Peigné la notion de "diversité » repose sur des catégorisations construites et réductrices (ethnies, langues, cultures, etc.) qui masquent de multiples hétérogénéités révélées par le travail distancié des chercheurs. La réflexion s'appuie sur deux cas («vignettes ») en contextes universitaires, extrêmement différents l'un de l'autre, qui montrent combien la notion de diversité repose sur des catégories homogénéisantes trop réductrices. Ainsi tous les étudiants chinois réels, en France, ne peuvent entrer dans la catégorie des « apprenants chinois » qui isole seulement quelques 
traits stéréotypés. Par exemple encore, la conception de la diversité dont se réclame l'Afrique du Sud, qui associe systématiquement sur un mode simplificateur une "langue " à une « ethnie », est déconstruite afin de mieux rendre compte, dans les départements de français, de l'hétérogénéité sociolinguistique des nouveaux publics enseignants et étudiants et de ses conséquences sur les objectifs curriculaires. C'est pourquoi, selon les auteurs, la diversité est à appréhender dans sa dimension qu'ils qualifient de diversitaire, c'est-à-dire complexe.

\section{Présentation des contributions de la partie varia}

Dans cette partie figurent deux articles qui ne sont pas sans rapport avec la thématique du dossier, même s'ils ne traitent pas d'enseignements universitaires francophones en milieux bi / plurilingues. En effet, dans sa contribution intitulée L'enseignement bilingue franco-italien en Italie et le diplôme binational Esabac: une coopération au carrefour d'enjeux politiques et didactiques, Hélène David s'efforce de montrer comment la création du diplôme binational franco-italien sanctionnant la fin des études secondaires (Esabac), créé en 2009 et mis en place en 2011, est le fruit de politiques linguistiques et éducatives initiées et légitimées aux niveaux européen et mondial, dont les acteurs aux niveaux national et local s'emparent pour servir chacun leurs intérêts propres. Hélène David nous donne un aperçu de l'analyse développée dans sa thèse, en s'appuyant sur un corpus de documents comprenant les préambules des accords de 2007 et 2009 ainsi que les programmes officiels de l'Esabac. À la fin de l'étude, la pérennité d'un tel dispositif est interrogée et, le cas échant, dans quel cadre. Autrement dit, sous prétexte d'éducation plurilingue et pluriculturelle n'a-t-on pas mis en place une filière bilingue qui cherche plutôt à se positionner sur le marché convoité de la formation des élites ?

Dans la dernière contribution intitulée L'influence $d u$ contexte sociolinguistique sur l'enseignement-apprentissage du français dans le Sud algérien. Le français, langue seconde ou étrangère? Le cas du Souf, Afaf Boudebia-Baala interroge le statut effectif du français dans la région du Souf (Sud-Est algérien), difficilement accessible par les colonisateurs en leur temps. En s'appuyant sur des questionnaires soumis à des enseignants du primaire puis complétés par des entretiens individuels et collectifs, elle catégorise les représentations exprimées par les enquêtés au sujet de la langue française, officiellement première langue étrangère enseignée à l'école primaire. Le français qui n'est pratiquement pas présent dans l'environnement social des élèves, que les parents ne maîtrisent absolument pas, dont le coefficient est faible par rapport à d'autres matières et qui fut la langue du colonisateur, est très mal perçu dans le milieu social en général et en particulier par les parents et les responsables éducatifs. En bref, le français ne suscite aucun intérêt bien qu'une légère évolution semble se dessiner depuis qu'un certain nombre d'étudiants soufis accède à l'université. Cette étude peut aussi se lire en écho à celle de Cortier, Kaaboub, Kherra et Benaoum pour comprendre concrètement l'origine des difficultés d'un enseignement francophone dans les filières scientifiques et techniques de l'enseignement supérieur.

\section{Pour finir}

À la lecture des articles du dossier thématique on constate tout d'abord que moins la légitimation d'un dispositif d'enseignement universitaire francophone en milieux bi / 
plurilingues est portée, au plus haut niveau institutionnel, par une politique linguistique articulée et explicite et moins sa mise en œuvre repose sur une action réfléchie, concertée et planifiée. Ainsi, en Ontario, au Canada, où le français est une des deux langues officielles, il nous est donné à voir un dispositif développé, le « régime d'immersion en français ", dont la mise en œuvre bénéficie de savoir-faire experts et multiples dans le domaine de la formation bi / plurilingue. En revanche, en Algérie où le français a officiellement le statut de langue étrangère bien qu'il fonctionne comme langue d'enseignement dans les facultés d'universités, les écoles et les instituts supérieurs des domaines scientifiques et techniques, les mises en œuvre de ces dispositifs ne reposent pas sur une ingénierie de formation dûment expérimentée et évaluée pour ce qui a trait à l'enseignement en français. Elles bénéficient tout au plus d'expériences singulières encore très récentes de pratiques d'enseignement-apprentissage du français en contexte universitaire bi / plurilingue, nécessairement innovantes certes (Mangiante \& Parpette, 2011), mais qui doivent être évaluées dans leur ensemble afin, éventuellement, d'être généralisées. Enfin, à Istanbul, à l'Université de Galatasaray, de même que dans les filières universitaires francophones à Bucarest et Sofia, où le français est une langue étrangère, non seulement officiellement mais aussi d'un point de vue sociolinguistique, la mise en œuvre de ces dispositifs nouveaux semble n'avoir bénéficié d'aucune réflexion approfondie pour ce qui concerne les difficultés d'un enseignement en français.

13 Si cette (dé)gradation n'est pas particulièrement étonnante, on peut remarquer que même dans le cas le plus favorable, sous l'angle sociolangagier, à la mise en place d'une formation tout n'est pas résolu au mieux lorsqu'il s'agit d'être attentif à la prise en compte et à la reconnaissance des répertoires langagiers des étudiants concernés. En Ontario, les dispositifs d'immersion ne favorisent pas la prise en compte de certains choix individuels, pourtant admis formellement par les institutions. Pour beaucoup d'universitaires encore, ne peuvent être reconnus comme francophones que ceux parmi les étudiants qui correspondent aux représentations stéréotypées (consonance du nom patronymique, origine géographique, etc.). En Afrique du Sud, les nouveaux publics d'étudiants et d'enseignants universitaires de français bouleversent les représentations habituelles et remettent implicitement en cause les choix élitistes traditionnels concernant les variétés langagières de français admises, les locuteurs légitimes, etc.

De fait, les légitimations se font, avant tout, en rapport avec le choix d'une variété de français normée de France ou du Québec dans le souci de la préservation de celle-ci mais sans celui de la prise en compte du répertoire langagier bi / plurilingue des étudiants. Ainsi, les mises en œuvre des dispositifs analysés portent avant tout sur l'enseignement dans une variété de français dont la maîtrise assure aux locuteurs une reconnaissance académique valorisante comme dans l'exemple du Caire, ou dans celui du Maroc. Seule l'étude sur le contexte algérien exprime la préoccupation du maintien des répertoires bi / plurilingues des étudiants, mais il faut comprendre, répétons-le, qu'il ne s'agit encore que d'une recherche-action expérimentale. 


\section{BIBLIOGRAPHIE}

Abécassis, F. (2006). «L'enseignement français en Égypte dans les années 1930 : les dévaluations contraintes d'un modèle ». In Cabanel, P. (dir.). Une France en Méditerranée. Écoles, langue et culture françaises. XIX ${ }^{e}-X X^{e}$ siècles. Paris : Éditions Créaphis. pp. 279-302.

Aksöy, E. (2007). « La francophonie en Turquie de l'Empire à nos jours ». Documents pour l'histoire du français langue étrangère ou seconde, $\mathrm{n}^{\circ}$ 38-39. pp. 57-66. Disponible en ligne. http:// dhfles.revues.org/138

Calvet, L-J. (2003). « Le plurilinguisme alexandrin ». In Bulot T. \& Messaoudi L. (dir.). Sociolinguistique urbaine (frontières et territoires). Cortil-Wodon : Éditions Modulaires Européennes. pp. 13-52.

Mangiante J.M. \& Parpette C. (2011). Le français sur objectif universitaire. Grenoble : PUG.

\section{NOTES}

1. Plurilinguisme: pratiques, représentations, acquisition, enseignement ( http:// www.projetpluri-l.org/).

2. Lire la constitution marocaine dans sa version de juin 2011, [http://www.sgg.gov.ma/ bo5952F.pdf?cle=42] et celle de l'Algérie [http://www.joradp.dz/hfr/Consti.htm].

3. Ce qui correspondait à l'organisation en millets dans l'Empire ottoman.

\section{RÉSUMÉS}

Le dossier thématique de ce numéro, issu des travaux de l'axe Éducation et Plurilinguismes : mises en perspective historiques et sociales du projet régional Pluri- $\mathrm{L}^{1}$ de la région des Pays de la Loire, est consacré à diverses légitimations et mises en œuvre d'enseignements universitaires francophones en milieux bi / plurilingues. À ce titre, nous souhaitons apporter des éclairages variés, tenant compte du statut des langues en présence, au sujet du développement d'une compétence bi / plurilingue à l'université, que ce soit du point de vue des acteurs concernés, des dispositifs conçus ou des difficultés rencontrées au moment de la réalisation. Une partie varia comprend deux études qui concernent l'une le niveau primaire et l'autre le niveau secondaire.

The thematic dossier of this issue stems from the work carried out by Éducation et Plurilinguismes: mises en perspective historiques et sociales a strand of the regional project Pluri- $\mathrm{L}^{1}$ (Pays de la Loire region), and is dedicated to diverse legitimations and implementations of Francophone university teaching in bi-plurilingual milieus. By taking into account the status of the languages involved, we would like to offer various perspectives on the development of a bi-plurilingual competence at the university be it from the point of view of the participants, the programmes 
put in place, or the challenges faced during their implementation. A varia section includes two studies that focus respectively on primary and secondary levels.

\section{INDEX}

Mots-clés : bi / plurilinguisme, contextes universitaires, français langue d'enseignement, politiques linguistiques

\section{AUTEURS}

\section{DANIELLE OMER}

Université du Maine, France

Danielle Omer est maître de conférences en sciences du langage à l'université du Maine. Ses activités de recherche en sociodidactique portent d'une part sur les écrits des étudiants dits natifs et non natifs en formation d'enseignants de FLE / $\mathrm{S}$ et d'autre part sur des contextes éducatifs passés plurilingues et francophones dans le Bassin méditerranéen, durant la période coloniale. Elle est membre du Projet Pluri-L (Plurilinguisme : pratiques, représentations, acquisition, enseignement) de la région des Pays de la Loire, responsable de l'axe : Éducation et Plurilinguismes : mises en perspective historiques et sociales.

Courriel : danielle.omer[at]univ-lemans.fr

Toile : http://www.projetpluri-l.org/

\section{FRÉDÉRIC TUPIN}

Université de la Réunion, France

Frédéric Tupin est professeur des universités en sciences de l'éducation à l'université de La Réunion. Ses travaux de recherche s'inscrivent dans une double perspective sociologique et sociolinguistique et ont pour objet les processus d'enseignement-apprentissage ainsi que l'analyse contextualisée des situations scolaires plurilingues et pluriculturelles. Une partie de ses activités de recherche s'inscrivent au sein du projet Pluri-L (Plurilinguisme : pratiques, représentations, acquisition, enseignement) de la région des Pays de la Loire, dont il est le porteur.

Courriel: frederic.tupin[at]univ-reunion.fr Toile: http://www.projetpluri-l.org/ 